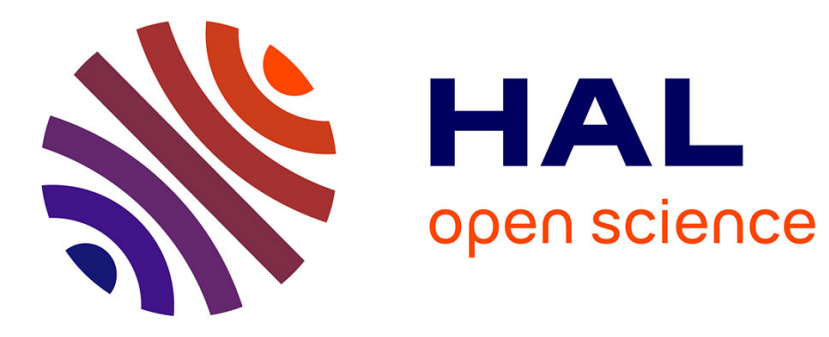

\title{
Cut-Free Display Calculi for Nominal Tense Logics
} Stéphane Demri, Rajeev Goré

\section{To cite this version:}

Stéphane Demri, Rajeev Goré. Cut-Free Display Calculi for Nominal Tense Logics. Automated Reasoning with Analytic Tableaux and Related Methods, International Conference, TABLEAUX '99, Saratoga Springs, NY, Neil Murray, Jun 1999, Saratoga Springs, United States. pp.155-170, 10.1007/3-540-48754-9_16. hal-03201530

\section{HAL Id: hal-03201530 https://hal.science/hal-03201530}

Submitted on 19 Apr 2021

HAL is a multi-disciplinary open access archive for the deposit and dissemination of scientific research documents, whether they are published or not. The documents may come from teaching and research institutions in France or abroad, or from public or private research centers.
L'archive ouverte pluridisciplinaire HAL, est destinée au dépôt et à la diffusion de documents scientifiques de niveau recherche, publiés ou non, émanant des établissements d'enseignement et de recherche français ou étrangers, des laboratoires publics ou privés. 


\title{
Cut-free Display Calculi for Nominal Tense Logics
}

\author{
Stéphane Demri ${ }^{1 \star}$ and Rajeev Goré ${ }^{2 \star \star}$ \\ 1 Laboratoire LEIBNIZ - C.N.R.S. \\ 46 av. Félix Viallet, 38000 Grenoble, France \\ demri@imag.fr \\ 2 Automated Reasoning Project and Dept. of Computer Science \\ Australian National University \\ ACT 0200 Canberra, Australia \\ rpg@arp.anu.edu.au
}

\begin{abstract}
We define cut-free display calculi for nominal tense logics extending the minimal nominal tense logic (MNTL) by addition of primitive axioms. To do so, we use a translation of MNTL into the minimal tense logic of inequality $\left(\mathrm{MTL}_{\neq}\right)$which is known to be properly displayable by application of Kracht's results. The rules of the display calculus $\delta$ MNTL for MNTL mimic those of the display calculus $\delta \mathrm{MTL}_{\neq}$for MTL $\neq$. Since $\delta$ MNTL does not satisfy Belnap's condition (C8), we extend Wansing's strong normalisation theorem to get a similar theorem for any extension of $\delta$ MNTL by addition of structural rules satisfying Belnap's conditions (C2)-(C7). Finally, we show a weak Sahlqvist-style theorem for extensions of MNTL, and by Kracht's techniques, deduce that these Sahlqvist extensions of $\delta$ MNTL also admit cut-free display calculi.
\end{abstract}

\section{Introduction}

Background: The addition of names (also called nominals) to modal logics has been investigated recently with different motivations; see e.g. [Orło84,PT85,Bla90]. A name is usually understood as an atomic proposition that holds true in a unique world of a Kripke model. Most of the time, the addition of names is intended to increase the expressive power of the initial logics. For instance, there is a tense formula with names that characterises the class of irreflexive frames [Bla93] although there is no such formula without names. Another remarkable breakthrough due to the inclusion of names is the ability to define the intersection operator (see e.g. [PT91]) although it is known that intersection is not modally definable in the standard modal language [GT75]. Adding the difference operator $[\neq]$, which allows access to worlds different from the current world, is another way to obtain names (see e.g. [Koy92,Rij92,Ven93]). As far as expressive power is concerned, adding $[\neq]$ is more powerful than adding only names: in [GG93], the relationships between names and $[\neq]$ are fully established with respect to definability. So most of the literature for modal logics with names concerns their

^ Visit to ARP supported by an Australian Research Council International Fellowship.

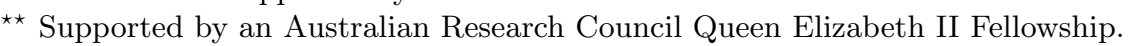


expressive power, decidability, complexity (see e.g. [Bla90,Rij93,GG93,PT91]) and Hilbert-style systems [Bla90,PT91,Ven93,Rij93].

Display Logic: Display Logic (abbreviated by DL) is a proof-theoretical framework due to Belnap [Bel82] that generalises the structural language of Gentzen's sequents by using multiple structural connectives instead of Gentzen's comma. Display calculi enjoy various nice properties. The first is that any display calculus that obeys eight simple conditions C1-C8 (see the appendix) also enjoys a cut-elimination theorem [Bel82]. The second is that, in the rules introducing logical connectives, the principal formula is alone as an antecedent or succedent, thereby giving a clear definition of the introduced connective. Consequently, interactions between logical connectives are reduced to a minimum. All of this is possible because any occurrence of a structure in a sequent can be displayed either as the entire antecedent or as the entire succedent of some sequent structurally equivalent to the initial sequent.

Our contribution: We define cut-free display calculi for two classes of extensions of the minimal nominal tense logic (MNTL) [Bla90], by addition of two types of primitive axioms in the sense of [Kra96]. These display calculi break (C8). We extend various results for displayed tense logics (including strong normalisation) from [Wan94,Kra96,Wan98] to nominals. Our main contribution is to show that Belnap's condition $\mathrm{C} 8$ can be weakened while preserving cut-elimination.

We first define the basic display calculus $\delta$ MNTL by using a natural translation from MNTL into MTL $\neq$, the minimal tense logic augmented with the difference operator. Indeed, $\mathrm{MTL}_{\neq}$is properly displayable in the sense of [Kra96] thanks to the Hilbert-style axiomatisation given in [Rij92] (see also [Seg81,Koy92]). The rules for $\delta$ MNTL mimic those of $\delta \mathrm{MTL}_{\neq}$, the display calculus for MTL $\neq$. We prove soundness of $\delta$ MNTL by showing that the rules preserve MNTL-validity. We prove completeness of $\delta$ MNTL by showing that $\delta$ MNTL can simulate the rules of the Hilbert-style calculus $\vdash_{\text {MNTL }}$ for MNTL given in [Bla90]. Cut-elimination cannot be proved via that proof because cut is needed to simulate the modus ponens rule.

An interesting (and at first glance very unpleasant) feature of $\delta$ MNTL is that it does not satisfy the condition (C8) [Bel82] which is crucial for the cut-elimination proofs from [Bel82,Wan98]. We show that the failure of (C8) is caused by the introduction rules for nominals and then show a limited cut-elimination theorem by observing that one of these rules is not really necessary. By appropriately modifying a proof from [Wan98], we then prove a strong normalisation theorem for any extension of $\delta$ MNTL obtained by the addition of structural rules satisfying the conditions $(\mathrm{C} 2)-(\mathrm{C} 7)$ from [Bel82] (condition (C8) only makes sense for logical rules). From a technical viewpoint, we have modified the definitions of parametric and principal moves to view a sequent in a proof as its equivalence class with respect to structural equivalence. Consequently, a display postulate inference in a proof does not add to the size of the proof. This can be generalised for any invertible structural rule with a single premiss.

We then have to make a connection between axiomatic extensions of $\vdash_{\text {MNTL }}$ and corresponding extensions of $\delta$ MNTL obtained by adding structural rules à la Kracht [Kra96]. Since $\delta$ MNTL is based upon $\delta \mathrm{MTL}_{\neq}$, we proceed via axiomatic 
extensions of $\vdash_{\neq}$, the Hilbert-style calculus for MTL $\neq$. Many such extensions of $\operatorname{MTL} \neq$ require the powerful (and sometimes redundant) irreflexivity rule (see e.g. [Gab81]) and, unfortunately, the corresponding rule in DL lacks various nice properties of standard display calculi. Although it is not always known when the irreflexivity rule is really needed, it is not needed in the axiomatisation $r_{\neq}$of $\mathrm{MTL}_{\neq}$. We therefore prove cut-elimination and completeness of $\delta$ MNTL with respect to primitive axiomatic extensions of $\vdash_{\mathrm{MTL} \neq}$ which do not require the irreflexivity rule, by backward translation (Theorem 10). These primitive axioms possibly contain the difference operator, which is foreign to MNTL.

Finally, although many extensions of MNTL are not canonical [Bla90], we show a weak Sahlqvist-style theorem for nominal tense logics. This allows us to define cut-free display calculi for any extension of $\vdash_{\text {MNTL }}$ by addition of another class of primitive axioms using only the language of MNTL, and hence without the difference operator. Furthermore, we can characterise the semantical extensions of MNTL which correspond to these calculi.

Related work: Existing proof systems for nominal tense logics [Bla90,Bla93] or for modal logics with the difference operator [Seg81,Koy92,Rij92,Ven93] are mostly Hilbert-style. And although the prefixed tableaux defined in [BD97] for several modal logics with the difference operator give decision procedures, a cut rule present in these calculi is not eliminable in many cases (for reasons similar to those that apply to calculi from [dM94]). Gentzen-style calculi for similarity logics with names have been defined in [Kon97] where the nominals play the rôle of prefixes in an elegant manner. These calculi contain no prefixed formulae as such since the language of the logic already contains names.

Our treatment of nominals in $\delta$ MNTL is different since we instead use the double nature of a nominal: as atomic proposition $i$ and as necessity formula $[\neq] \neg i$. In that sense, it is similar to the treatment of atomic propositions in display calculi for intuitionistic logic in [Gor95]. In [Bla98,Dem99], sequent calculi for nominal tense logics are given in which the nominals roughly play the role of labels. Cut-free display calculi have also been defined for substructural logics (see e.g. [Gor98]) and for modal and polymodal logics [Wan94,Kra96]. In [Ven93], a Sahlqvist theorem for tense logics with the difference operator has been established for calculi with the irreflexivity rule (see also [Rij93]).

Plan of the paper: In Section 2, we recall the definitions of the logics under study [Bla90,Rij92,Ven93]. In Section 3, we define the cut-free display calculus $\delta$ MNTL for MNTL, show its completeness and prove a (weak) cut-elimination theorem. In Section 4, we prove a strong normalisation theorem for any reasonable extension of $\delta$ MNTL although $\delta$ MNTL does not satisfy Belnap's condition (C8). In Section 5 we establish a weak Sahlqvist-style theorem and, by using [Kra96], define cut-free display calculi for extensions of MNTL. Space limits preclude detailed proofs, but these can be found in the full version [DG98a]. Belnap's eight conditions, and our weaker version of (C8), can be found in the appendix. 


\section{Nominal Tense Logics}

Given a set $\mathrm{PRP}=\left\{\mathrm{p}_{0}, \mathrm{p}_{1}, \mathrm{p}_{2}, \ldots\right\}$ of atomic propositions and a set $\mathrm{NOM}=$ $\left\{\mathbf{i}_{0}, i_{1}, \ldots\right\}$ of names, the formulas $\phi \in \operatorname{NTL}(G, H,[\neq])$ are inductively defined as follows for $\mathrm{p}_{j} \in \mathrm{PRP}, \mathrm{i}_{k} \in \mathrm{NOM}$ :

$\phi::=\top|\perp| \mathrm{p}_{j}\left|\mathrm{i}_{k}\right| \phi_{1} \wedge \phi_{2}\left|\phi_{1} \vee \phi_{2}\right| \phi_{1} \Rightarrow \phi_{2}|\neg \phi| H \phi|G \phi|[\neq] \phi$

Standard abbreviations include $\Leftrightarrow,\langle\neq\rangle, F, P$. For instance $F \phi \stackrel{\text { def }}{=} \neg G \neg \phi$.

For any sequence $\overline{\mathrm{OP}}$ from $\{H, G,[\neq]\}$, we write NTL $(\overline{\mathrm{OP}})$ to denote the fragment of $\operatorname{NTL}(G, H,[\neq])$ with the unary modal operators from $\overline{\mathrm{OP}}$. Similarly, TL $(\overline{\mathrm{OP}})$ denotes the fragment of NTL $(\overline{\mathrm{OP}})$ with no names. In the rest of the paper, we study logics whose languages are strict fragments of $\operatorname{NTL}(G, H,[\neq])$ (the whole language contains all that we need in the paper). For any $\phi \in \operatorname{NTL}(G, H,[\neq])$, we write $d g(\phi)$ to denote the degree of $\phi$ : that is the number of occurrences of members of $\mathrm{PRP} \cup \mathrm{NOM} \cup\{\top, \perp\} \cup\{\neg, \wedge, \vee, \Rightarrow, G, H,[\neq]\}$. For instance $d g\left(\perp \Rightarrow\left(\mathrm{i}_{0} \vee \neg \mathrm{p}_{1}\right)\right)=6$.

A modal frame $(W, R)$ is a pair where $W$ is a non-empty set and $R$ is a binary relation over $W$, with $R^{-1}$ the converse of $R$. We write $F r$ for the set of all modal frames and use $R(w) \stackrel{\text { def }}{=}\{v \in W:(w, v) \in R\}$. A model is a triple $(W, R, m)$ such that $(W, R)$ is a frame, $\mathcal{P}(W)$ is the set of all subsets of $W$, and $m$ is a mapping $m: \operatorname{PRP} \cup \mathrm{NOM} \rightarrow \mathcal{P}(W)$ where for $i \in$ NOM, $m(i)$ is a singleton.

Let $\mathcal{M}=(W, R, m)$ be a model and $w \in W$. As usual, the formula $\phi$ is satisfied by the world $w \in W$ in $\mathcal{M} \stackrel{\text { def }}{\Leftrightarrow} \mathcal{M}, w=\phi$ where the satisfaction relation $\models$ is inductively defined as follows:

$$
\begin{aligned}
& \mathcal{M}, w=\mathrm{p} \stackrel{\text { def }}{\Leftrightarrow} w \in m(\mathrm{p}), \text { for every } \mathrm{p} \in \mathrm{PRP} \cup \mathrm{NOM} \\
& \mathcal{M}, w=G \phi \stackrel{\text { def }}{\Leftrightarrow} \text { for every } v \in R(w), \mathcal{M}, v \models \phi ; \\
& \mathcal{M}, w=H \phi \stackrel{\text { def }}{\Leftrightarrow} \text { for every } v \in R^{-1}(w), \mathcal{M}, v=\phi ; \\
& \mathcal{M}, w=[\neq] \phi \stackrel{\text { def }}{\Leftrightarrow} \text { for every } v \neq w, \mathcal{M}, v=\phi .
\end{aligned}
$$

We omit the standard conditions for the propositional connectives and the logical constants. A formula $\phi$ is true in a model $\mathcal{M}$ (written $\mathcal{M} \models \phi) \stackrel{\text { def }}{\Leftrightarrow}$ for every $w \in W, \mathcal{M}, w=\phi$. A formula $\phi$ is true in a frame $\mathcal{F}$ (written $\mathcal{F} \models \phi$ ) $\stackrel{\text { def }}{\Leftrightarrow} \phi$ is true in every model based on $\mathcal{F}$. By a logic $\mathcal{L}$ we understand a pair $\langle\mathrm{L}, \mathcal{C}\rangle$ consisting of a language $\mathrm{L} \subseteq \operatorname{NTL}(H, G,[\neq])$ and a nonempty set of frames $\mathcal{C} \subseteq$ Fr. A formula $\phi \in \mathrm{L}$ is $\mathcal{L}$-valid $\stackrel{\text { def }}{\Leftrightarrow} \phi$ is true in all the models based on the frames in $\mathcal{C}$. A formula $\phi \in \mathrm{L}$ is $\mathcal{L}$-satisfiable $\stackrel{\text { def }}{\Leftrightarrow} \neg \phi$ is not $\mathcal{L}$-valid.

The minimal nominal tense logic is MNTL $\stackrel{\text { def }}{=}\langle\operatorname{NTL}(H, G), F r\rangle$. The minimal tense logic of inequality is $\mathrm{MTL}_{\neq} \stackrel{\text { def }}{=}\langle\mathrm{TL}(G, H,[\neq]), F r\rangle$. Moreover, for any formula $\phi$ of some language $\mathrm{L} \subseteq \operatorname{NTL}(H, G,[\neq])$ with names [resp. without names], we write $\mathrm{NTL}_{\phi}[$ resp. TL $\phi]$ to denote the logic $\langle\mathrm{L},\{\mathcal{F} \in F r: \mathcal{F} \models \phi\}\rangle$.

By a universal modality [resp. existential modality] $\sigma$, we mean a (possibly empty) finite sequence of elements from $\{G, H\}$ [resp. $\{F, P\}]$. We write $\vdash_{\neq}$to denote the axiomatic system defined in [Rij93, pp. 36-37] for $\mathrm{MTL}_{\neq}$. We write $\vdash_{\text {MNTL }}$ for the smallest subset of $\operatorname{NTL}(G, H)$ closed under modus ponens, closed under necessitation for $G$ and $H$, and containing every formula of the form 
- the tautologies of the propositional calculus;

- $(G(\phi \Rightarrow \psi) \wedge G \phi) \Rightarrow G \psi ;(H(\phi \Rightarrow \psi) \wedge H \phi) \Rightarrow H \psi ; \phi \Rightarrow H F \phi ; \phi \Rightarrow G P \phi ;$

- $\mathbf{i} \wedge \phi \Rightarrow \sigma(i \Rightarrow \phi)$ where $i \in$ NOM and $\sigma$ is any universal modality.

We write $\vdash \psi$ to mean that $\psi$ is derivable in the Hilbert-style calculus $\vdash$. We write $\vdash+\phi$ to denote the minimal extension of the axiomatic system $\vdash$ by adding all formulae of the form $\phi$ (thus $\phi$ is just an axiom schema).

Theorem 1. [Bla90] Any $\phi \in \operatorname{NTL}(H, G)$ is MNTL-valid iff $\vdash_{\text {MNTL }} \phi$.

\section{A Display Calculus for MNTL}

As stated previously there are numerous existing display calculi. We use Wansing's [Wan94] formulation since it is tailored to modal logics. On the structural side, we have the structural connectives $*$ (unary), ○ (binary), $I$ (nullary), • (unary) and $\bullet \neq$ (unary). A structure $\mathrm{X} \in \operatorname{struc}(\delta \mathrm{MNTL})$ is inductively defined as

$$
\mathrm{X}::=\phi|* \mathrm{X}| \mathrm{X}_{1} \circ \mathrm{X}_{2}|I| \bullet \mathrm{X} \mid \bullet \neq \mathrm{X}
$$

for $\phi \in \operatorname{NTL}(G, H)$. A logical interpretation of the structural connectives can be found in the proof of the forthcoming Theorem 3. A sequent is defined as an expression of the form $\mathrm{X} \vdash \mathrm{Y}$ with $\mathrm{X}$ the antecedent and $\mathrm{Y}$ the succedent. For any finite set $S$ of structures, we write $\operatorname{NOM}(S)$ for the set of names from NOM that occur in $S$. We write $\varphi_{S}$ to denote the formula (in $\operatorname{TL}(G, H,[\neq])$ ) below:

$$
\bigwedge_{\mathrm{i}_{k} \in \operatorname{NOM}(S)}\left(\mathrm{p}_{2 \times k+1} \wedge[\neq] \neg \mathrm{p}_{2 \times k+1}\right) \vee\langle\neq\rangle\left(\mathrm{p}_{2 \times k+1} \wedge[\neq] \neg \mathrm{p}_{2 \times k+1}\right)
$$

The rules of $\delta$ MNTL are those in Figures 1-5.

The display postulates (reversible rules) in Figure 2 deal with the manipulation of structural connectives. In what follows, we write

$$
\frac{s}{s^{\prime}}(d p)
$$

to denote that the sequent $s^{\prime}$ is obtained from the sequent $s$ by an unspecified finite number (possibly zero) of applications of display postulates.
(Id) $\mathrm{p} \vdash \mathrm{p}$
$\left(\operatorname{Id}^{\prime}\right) \quad i \vdash i$
$\frac{\mathrm{X} \vdash \phi \quad \phi \vdash \mathrm{Y}}{\mathrm{X} \vdash \mathrm{Y}}(\mathrm{cut})$

Fig. 1. Fundamental logical axioms and cut rule 


$$
\begin{aligned}
& \frac{\mathrm{X} \circ \mathrm{Y} \vdash \mathrm{Z}}{\overline{\mathrm{X} \vdash \mathrm{Z} \circ * \mathrm{Y}}} \quad \overline{\overline{\mathrm{Y} \vdash * \mathrm{X} \circ \mathrm{Z}}} \quad \overline{\overline{\mathrm{X} \circ * \mathrm{Z} \vdash \mathrm{Y}}} \quad \frac{\overline{\overline{\mathrm{X}} \vdash \mathrm{Y} \circ \mathrm{Y} \circ \mathrm{X} \vdash \mathrm{Z}}}{\overline{* \mathrm{Y}}} \\
& \frac{* \mathrm{X} \vdash \mathrm{Y}}{\overline{* \mathrm{Y} \vdash \mathrm{X}}} \quad \frac{\mathrm{X} \vdash * \mathrm{Y}}{\overline{\mathrm{Y} \vdash * \mathrm{X}}} \quad \frac{* * \mathrm{X} \vdash \mathrm{Y}}{\overline{\mathrm{X} \vdash \mathrm{Y}}} \quad \frac{\mathrm{X} \vdash * * \mathrm{Y}}{\overline{\mathrm{X} \vdash \mathrm{Y}}} \quad \frac{\mathrm{X} \vdash \bullet \neq \mathrm{Y}}{\bullet_{\neq} \mathrm{X} \vdash \mathrm{Y}} \quad \frac{\mathrm{X} \vdash \bullet \mathrm{Y}}{\bullet \mathrm{X} \vdash \mathrm{Y}}
\end{aligned}
$$

Fig. 2. Display postulates

In any structure $\mathrm{Z}$, the structure $\mathrm{X}$ occurs negatively [resp. positively] $\stackrel{\text { def }}{\Leftrightarrow} \mathrm{X}$ occurs in the scope of an odd number [resp. an even number] of occurrences of * [Bel82]. In a sequent $\mathrm{V} \vdash \mathrm{W}$, an occurrence of $\mathrm{X}$ is an antecedent part [resp. succedent part] $\stackrel{\text { def }}{\Leftrightarrow}$ it occurs positively in $\mathrm{V}$ [resp. negatively in $\mathrm{W}$ ] or it occurs negatively in $\mathrm{W}$ [resp. positively in $\mathrm{V}$ ] [Bel82]. Two sequents $\mathrm{X} \vdash \mathrm{Y}$ and $\mathrm{X}^{\prime} \vdash \mathrm{Y}^{\prime}$ are said to be structurally equivalent $\stackrel{\text { def }}{\Leftrightarrow}$ there is a derivation of the first sequent from the second (and vice-versa) using only the display postulates.

Theorem 2. [Bel82] For every sequent $\mathrm{V} \vdash \mathrm{W}$ and every antecedent [resp. succedent] part $\mathrm{X}$ of $\mathrm{V} \vdash \mathrm{W}$, there is a structurally equivalent sequent $\mathrm{X} \vdash \mathrm{Y}$ [resp. $\mathrm{Y} \vdash \mathrm{X}$ ] that has $\mathrm{X}$ (alone) as its antecedent [resp. succedent]. $\mathrm{X}$ is said to be displayed in $\mathrm{X} \vdash \mathrm{Y}[$ resp. $\mathrm{Y} \vdash \mathrm{X}]$.

A structural rule contains only structural connectives and structure variables like X, Y, Z. Following [Kra96], a formula is said to be primitive $\stackrel{\text { def }}{\Leftrightarrow}$ it is of the form $\phi \Rightarrow \psi$ where both $\phi$ and $\psi$ are built only from PRP $\cup\{\top\}$ with the help of $\wedge, \vee, F, P$ and $\langle\neq\rangle$, and such that $\phi$ contains each atomic proposition at most once. The rules in Figure 5 are translations of primitive axioms from the axiomatisation $\vdash_{\neq}$of $\mathrm{MTL}_{\neq}$[Rij93] into structural rules following [Kra96]. Thus $\bullet \neq$ is implicitly associated with the pair $\langle[\neq],\langle\neq\rangle\rangle$ of dual modal operators (since $\neq$ is symmetric), and $\bullet$ is associated with the pair of residuated operators $\langle G, P\rangle$.

$$
\begin{aligned}
& \overline{I \vdash \top}(\vdash \top) \frac{I \vdash \mathrm{X}}{\top \vdash \mathrm{X}}(\top \vdash) \frac{\mathrm{X} \vdash I}{\mathrm{X} \vdash \perp}(\vdash \perp) \frac{}{\perp \vdash I}(\perp \vdash) \frac{\mathrm{X} \vdash * \phi}{\mathrm{X} \vdash \neg \phi}(\vdash \neg) \frac{* \phi \vdash \mathrm{X}}{\neg \phi \vdash \mathrm{X}}(\neg \vdash) \\
& \frac{\mathrm{X} \circ \phi \vdash \psi}{\mathrm{X} \vdash \phi \Rightarrow \psi}(\vdash \Rightarrow) \frac{\mathrm{X} \vdash \phi \psi \vdash \mathrm{Y}}{\phi \Rightarrow \psi \vdash * \mathrm{X} \circ \mathrm{Y}}(\Rightarrow \vdash) \frac{\mathrm{X} \vdash \phi \mathrm{Y} \vdash \psi}{\mathrm{X} \circ \mathrm{Y} \vdash \phi \wedge \psi}(\vdash \wedge) \frac{\phi \circ \psi \vdash \mathrm{X}}{\phi \wedge \psi \vdash \mathrm{X}}(\wedge \vdash) \\
& \frac{\mathrm{X} \vdash \phi \circ \psi}{\mathrm{X} \vdash \phi \vee \psi}(\vdash \vee) \frac{\phi \vdash \mathrm{X} \psi \vdash \mathrm{Y}}{\phi \vee \psi \vdash \mathrm{X} \circ \mathrm{Y}}(\vee \vdash) \frac{\phi \vdash \mathrm{X}}{G \phi \vdash \bullet \mathrm{X}}(G \vdash) \frac{\mathrm{X} \vdash \bullet \phi}{\mathrm{X} \vdash G \phi}(\vdash G) \\
& \frac{\phi \vdash \mathrm{X}}{H \phi \vdash * \bullet * \mathrm{X}}(H \vdash) \frac{\mathrm{x} \vdash * \bullet * \phi}{\mathrm{X} \vdash H \phi}(\vdash H) \quad \frac{\mathrm{i} \vdash \bullet * \mathrm{X}}{\mathrm{X} \vdash \mathrm{i}}(\vdash \mathrm{i}) \quad \frac{\mathrm{X} \vdash \mathrm{i}}{\mathrm{i} \vdash \bullet_{\neq *} \mathrm{X}}(\mathrm{i} \vdash)
\end{aligned}
$$

Fig. 3. Operational rules 


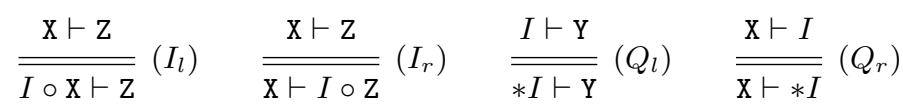

$$
\begin{aligned}
& \frac{\mathrm{X} \vdash \mathrm{Z}}{\mathrm{Y} \circ \mathrm{X} \vdash \mathrm{Z}}\left(\text { weak }_{l}\right) \frac{\mathrm{X} \vdash \mathrm{Z}}{\mathrm{X} \circ \mathrm{Y} \vdash \mathrm{Z}}\left(\text { weak }_{r}\right) \frac{\mathrm{X}_{1} \circ\left(\mathrm{X}_{2} \circ \mathrm{X}_{3}\right) \vdash \mathrm{Z}}{\overline{\left(\mathrm{X}_{1} \circ \mathrm{X}_{2}\right) \circ \mathrm{X}_{3} \vdash \mathrm{Z}}}\left(\text { assoc }_{l}\right) \\
& \frac{\mathrm{Z} \vdash \mathrm{X}_{1} \circ\left(\mathrm{X}_{2} \circ \mathrm{X}_{3}\right)}{\overline{\mathrm{Z} \vdash\left(\mathrm{X}_{1} \circ \mathrm{X}_{2}\right) \circ \mathrm{X}_{3}}}\left(\text { assoc }_{r}\right) \\
& \frac{\mathrm{Y} \circ \mathrm{X} \vdash \mathrm{Z}}{\mathrm{X} \circ \mathrm{Y} \vdash \mathrm{Z}}\left(\operatorname{com}_{l}\right) \frac{\mathrm{Z} \vdash \mathrm{Y} \circ \mathrm{X}}{\mathrm{Z} \vdash \mathrm{X} \circ \mathrm{Y}}\left(\operatorname{com}_{r}\right) \frac{\mathrm{X} \circ \mathrm{X} \vdash \mathrm{Y}}{\mathrm{X} \vdash \mathrm{Y}}\left(\operatorname{contr}_{l}\right) \frac{\mathrm{Y} \vdash \mathrm{X} \circ \mathrm{X}}{\mathrm{Y} \vdash \mathrm{X}}\left(\operatorname{contr}_{r}\right) \\
& \frac{I \vdash \mathrm{X}}{\bullet I \vdash \mathrm{X}}\left(n e c_{G}^{l}\right) \frac{\mathrm{X} \vdash I}{\mathrm{X} \vdash \bullet I}\left(n e c_{G}^{r}\right) \quad \frac{I \vdash \mathrm{X}}{\bullet \neq I \vdash \mathrm{X}}\left(n e c^{l}\right) \frac{\mathrm{X} \vdash I}{\mathrm{X} \vdash \bullet \neq I}\left(n e c^{r}\right)
\end{aligned}
$$

Fig. 4. Other basic structural rules

$$
\begin{aligned}
& \frac{\mathrm{X} \vdash \mathrm{Y} \quad * \bullet \neq * \mathrm{X} \vdash \mathrm{Y}}{* \bullet \neq \bullet \neq * \mathrm{X} \vdash \mathrm{Y}} \text { (alio) } \quad \frac{* \bullet \neq *(\mathrm{Z} \circ * \bullet \neq * \mathrm{X}) \vdash \mathrm{Y}}{\mathrm{X} \circ * \bullet \neq * \mathrm{Z} \vdash \mathrm{Y}} \text { (sym) } \\
& \frac{\mathrm{X} \vdash \mathrm{Y} \quad * \bullet \neq * \mathrm{X} \vdash \mathrm{Y}}{* \bullet * \mathrm{X} \vdash \mathrm{Y}}(\text { uni } 1) \quad \frac{\mathrm{X} \vdash \mathrm{Y} \quad * \bullet \neq * \mathrm{X} \vdash \mathrm{Y}}{\bullet \mathrm{X} \vdash \mathrm{Y}}(\text { uni } 2)
\end{aligned}
$$

Fig. 5. Other structural rules

An easy way to understand the way the rules $(i \vdash)$ and $(\vdash \mathbf{i})$ in Figure 3 work is to observe that the formula $i \Leftrightarrow[\neq] \neg i$ from the language $\operatorname{NTL}([\neq])$ is valid in any Kripke model. Thus, the rules $(i \vdash)$ and $(\vdash \mathbf{i})$ use the intensional nature of a name whereas the fundamental axiom $i \vdash i$ uses its atomic nature.

Theorem 3. (soundness) If $I \vdash \phi$ is derivable in $\delta$ MNTL, then $\phi$ is MNTL-valid.

Proof. Consider maps $a$ and $s$ from $\operatorname{struc}(\delta$ MNTL $)$ to $\operatorname{TL}(H, G,[\neq])$ as below: 


$$
\begin{aligned}
& a \text { and } s \text { are homomorphic for } \neg, \wedge, \vee, \Rightarrow, H \text { and } G \\
& \text { for every } \mathrm{p}_{j} \in \mathrm{PRP}, a\left(\mathrm{p}_{j}\right) \stackrel{\text { def }}{=} s\left(\mathrm{p}_{j}\right) \stackrel{\text { def }}{=} \mathrm{p}_{2 \times j} \\
& \text { for every } \mathbf{i}_{k} \in \mathrm{NOM}, a\left(\dot{i}_{k}\right) \stackrel{\text { def }}{=} s\left(\mathbf{i}_{k}\right) \stackrel{\text { def }}{=} \mathrm{p}_{2 \times k+1} \wedge[\neq] \neg \mathrm{p}_{2 \times k+1} \\
& a(\top) \stackrel{\text { def }}{=} s(T) \stackrel{\text { def }}{=} \top \quad a(\perp) \stackrel{\text { def }}{=} s(\perp) \stackrel{\text { def }}{=} \perp
\end{aligned}
$$

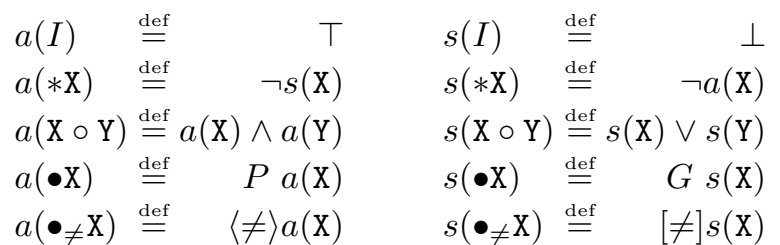

By induction on the length of the given derivation of $\mathrm{X} \vdash \mathrm{Y}$, we can show that if $\mathrm{X} \vdash \mathrm{Y}$ is derivable in $\delta \mathrm{MNTL}$, then $\varphi_{\{\mathrm{X}, \mathrm{Y}\}} \Rightarrow(a(\mathrm{X}) \Rightarrow s(\mathrm{Y}))$ is $\mathrm{MTL}_{\neq}$-valid. Furthermore, for any $\phi \in \operatorname{NTL}(\mathrm{H}, \mathrm{G}), \phi$ is MNTL-valid iff $a(\phi)$ is MTL $_{\neq}$-valid iff $s(\phi)$ is $\mathrm{MTL}_{\neq}$-valid iff $\varphi_{\{\phi\}} \Rightarrow a(\phi)$ is $\mathrm{MTL}_{\neq}$-valid iff $\varphi_{\{\phi\}} \Rightarrow s(\phi)$ is $\mathrm{MTL}_{\neq}$-valid. In particular, if $I \vdash \phi$ is derivable in $\delta$ MNTL, then $\varphi_{\{\phi\}} \Rightarrow(a(I) \Rightarrow s(\phi))$ (i.e. $T \Rightarrow s(\phi))$ is $\mathrm{MTL}_{\neq}$-valid and hence $\phi$ is MNTL-valid.

Next, we give a completeness proof of $\delta$ MNTL using the system $\vdash_{\text {MNTL }}$.

Lemma 1. Let $\mathrm{X} \vdash \mathrm{Y}$ and $\mathrm{X}^{\prime} \vdash \mathrm{Y}^{\prime}$ be sequents such that $\mathrm{X}^{\prime} \vdash \mathrm{Y}^{\prime}$ can be obtained from $\mathrm{X} \vdash \mathrm{Y}$ by replacing some occurrences of $* \bullet \neq * \mathrm{Z}$ by $\bullet \neq \mathrm{Z}$ and by replacing some occurrences of $\bullet \neq \mathrm{W}$ by $* \bullet * \mathrm{~W}$. Then, any display calculus $\delta$ containing the display postulates from Figure 2, (sym), ( contr $\left._{r}\right),\left(\right.$ weak $\left._{r}\right)$ and $\left(\right.$ weak $\left._{l}\right)$ satisfies: $\mathrm{X} \vdash \mathrm{Y}$ is derivable [resp. has a cut-free proof] in $\delta$ iff $\mathrm{X}^{\prime} \vdash \mathrm{Y}^{\prime}$ is derivable [resp. has a cut-free proof] in $\delta$.

Lemma 1 is unsurprising since (sym) corresponds to the axiom schema $F \phi \Leftrightarrow$ $P \phi$ characterising symmetry. However, Lemma 1 is purely syntactic.

Theorem 4. (completeness) If $\vdash_{\text {MNTL }} \phi$, then $I \vdash \phi$ is derivable in $\delta$ MNTL.

The proof of Theorem 4 relies only on the completeness of $\delta K t$ [Kra96] and on the derivability of the axiom schema i $\wedge \phi \Rightarrow \sigma(\mathrm{i} \Rightarrow \phi)$. Moreover, it highlights how the rules (uni1), (uni2), (sym) and (alio) are needed to get completeness. In what follows, we write

$$
\vdots \Pi
$$

to denote that the sequent $s$ has a proof $\Pi$ in $\delta$ MNTL.

Proof. (sketch) The proof is by induction on the length of the derivation of $\phi$ in $\vdash_{\text {MNTL }}$. Actually, most of the cases have been already proved in [Wan94,Kra96,Wan98]. It remains to show that $I \vdash \mathrm{i} \wedge \phi \Rightarrow \sigma(\mathrm{i} \Rightarrow \phi)$ is derivable in $\delta$ MNTL where $i \in \operatorname{NOM}, \phi \in \operatorname{NTL}(H, G)$ and $\sigma$ is a (possibly empty) finite sequence of elements from $\{H, G\}$. To do so, we prove by induction on the length of $\sigma$ that i $\circ \phi \vdash \sigma(i \Rightarrow \phi)$ and $\bullet \neq(\mathbf{i} \circ \phi) \vdash \sigma(i \Rightarrow \phi)$ are derivable in $\delta$ MNTL (see [DG98a]). 
Unfortunately, as shown shortly in Example 1, the rules $(\vdash \mathrm{i})$ and $(i \vdash$ ) from $\delta$ MNTL do not satisfy (C8), so we cannot prove cut-elimination using [Bel82,Wan98]. However, $\delta$ MNTL minus the rule $\left(\vdash\right.$ i), say $\delta^{-}$MNTL, obeys (C1)(C8) since, in $\delta^{-}$MNTL, i can be a succedent principal formula only in the fundamental axiom $i \vdash i$. Belnap's cut-elimination proof applies and therefore $\mathrm{X} \vdash \mathrm{Y}$ has a proof in $\delta^{-}$MNTL iff $\mathrm{X} \vdash \mathrm{Y}$ has a cut-free proof in $\delta^{-}$MNTL. A similar "trick" is used in [Gor95]. Surprisingly, the proof of Theorem 4 also shows that if $\vdash_{\text {MNTL }} \phi$, then $I \vdash \phi$ is derivable in $\delta^{-}$MNTL since the $(\vdash i)$-rule is simply not used. Consequently,

Theorem 5. (weak cut-elimination) If $\vdash_{\text {MNTL }} \phi$, then $I \vdash \phi$ has a cut-free proof in $\delta^{-}$MNTL.

Whether $\delta$ MNTL enjoys cut-elimination is still open at this stage of the paper since all the provable sequents $\mathrm{X} \vdash \mathrm{Y}$ are not necessarily of the form $I \vdash \phi$. Moreover, Theorem 5 does not guarantee that any reasonable extension of $\delta$ MNTL enjoys cut-elimination. In the next section we extend Wansing's strong normalisation theorem to $\delta$ MNTL in such a way that any extension of $\delta$ MNTL by addition of structural rules satisfying $(\mathrm{C} 2)-(\mathrm{C} 7)$ also satisfies the strong normalisation theorem (condition (C8) is relevant only for logical rules).

\section{A Strong Normalisation Theorem}

A very important feature of the proof-theoretical framework DL is the existence of a very general cut-elimination theorem [Bel82]. Indeed, any display calculus satisfying the conditions (C2)-(C8) [Bel82] admits cut-elimination. In [Wan98], such a result is strengthened by proving that any classical modal display calculus defined from [Kra96] for a properly displayable classical modal logic [Kra96] admits a strong normalisation theorem: that is, the process of cut-elimination terminates for any sequence of the reduction steps to be defined shortly. Similar theorems exist for numerous formal systems such as for example those for typed $\lambda$-terms (see e.g. [TS96]).

Unfortunately $\delta$ MNTL does not satisfy (C8) recalled below (see e.g. [Wan98]):

(C8) If there are inferences $\mathcal{I}_{1}$ and $\mathcal{I}_{2}$ with respective conclusions $\mathrm{X} \vdash \phi$ and $\phi \vdash \mathrm{Y}$ with $\phi$ principal in both inferences, and if cut is applied to obtain $\mathrm{X} \vdash \mathrm{Y}$, then either $\mathrm{X} \vdash \mathrm{Y}$ is identical to one of $\mathrm{X} \vdash \phi$ and $\phi \vdash \mathrm{Y}$; or there is a derivation of $\mathrm{X} \vdash \mathrm{Y}$ from the premisses of $\mathcal{I}_{1}$ and $\mathcal{I}_{2}$ in which every cut-formula of any application of cut is a proper subformula of $\phi$.

Example 1. Consider the proof,

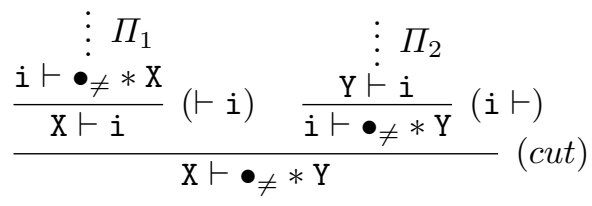


Since $i$ does not have proper subformulae, $\delta$ MNTL does not satisfy (C8) which is absolutely crucial in the proofs of cut-elimination in [Bel82,Wan98]. However, $\delta$ MNTL enjoys a (weak) cut-elimination theorem (see Theorem 5).

At first sight, C4 also seems to be violated since an inference of $(i \vdash)$ [resp. $(\vdash i)$ ] changes the displayed antecedent [resp. succedent] part occurrence of $i$ in the conclusion into a succedent [resp. antecedent] part occurrence in the premiss. However, all is well, for the occurrences of a name in some $(i \vdash)$-rule [resp. $(\vdash$ i)-rule] inference are not parameters since they are not substructures of some structure obtained by instantiating some structure variable.

We now show that any reasonable extension of $\delta$ MNTL admits a strong normalisation theorem by adapting arguments from [Wan98]. By a reasonable extension, we mean a calculus $\delta$ obtained from $\delta$ MNTL by addition of structural rules that satisfy the conditions (C2)-(C7) (see e.g. [Bel82,Kra96,Wan98]).

As usual, our strong normalisation theorem is relative to a given reduction concept. Indeed, we shall define legitimate moves that define the authorised reductions. Basically, each reduction removes a cut at the cost of cuts of lesser rank, or permutes a cut with a rule application in one of its premisses or replaces a cut by a cut of the same rank but decreases the number of significant inferences. In the rest of the section, $\delta$ is assumed to be reasonable.

The reduction process consists of SE-principal moves and parametric moves. First, let us recall that in DL, every structure occurrence in an inference $\mathcal{I}$ is called a constituent of $\mathcal{I}$. Constituents of an inference $\mathcal{I}$ are congruent $\stackrel{\text { def }}{\Leftrightarrow}$ they occupy similar positions in occurrences of structures assigned to the same structure variable.

Definition 1. In the proof $\Pi_{1}$ from Definition 2 below left, the congruence class of $\phi$ is the smallest set $Q_{\phi}$ of occurrences of $\phi$ in $\Pi_{1}$ such that

- the displayed occurrence of $\phi$ in $\mathrm{X} \vdash \phi$ is in $Q_{\phi}$;

- for every inference $\mathcal{I}$ in $\Pi_{1}$, each constituent of a premiss of $\mathcal{I}$ which is congruent (w.r.t. $\mathcal{I})$ to a constituent of the conclusion of $\mathcal{I}$ already in $Q_{\phi}$, is in $Q_{\phi}$.

$Q_{\phi}$ can be viewed as a finite tree of occurrences of $\phi . A$ path in the tree defined by $Q_{\phi}$ is a maximal finite sequence $\phi_{\text {occ }_{1}}, \ldots, \phi_{\text {occ }_{l}}(l \geq 1)$ of elements of $Q_{\phi}$ such that for $k \in\{1, \ldots, l-1\}, \phi_{o c c_{k}}$ is congruent to $\phi_{o c c_{k+1}}$ for some inference.

Definition 2. In the proof $\Pi$ below left, $\phi$ is said to be SE-principal ('principal modulo structural equivalence') in $\mathrm{X} \vdash \phi \stackrel{\text { def }}{\Leftrightarrow}$ the subproof $\Pi_{1}$ is of the form $\Pi_{1}^{\prime}$ shown below right, $\phi$ is principal in the instance of rule $(r u)$ and the two occurrences of $\phi$ in $\mathrm{X}^{\prime} \vdash \phi$ and $\mathrm{x} \vdash \phi$ in $\Pi_{1}^{\prime}$ belong to the same congruence class:

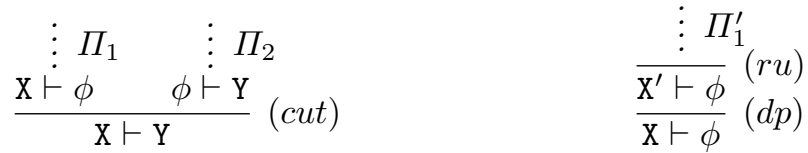

We use an analogous definition for $\phi$, SE-principal in $\phi \vdash \mathrm{Y}$. 
Consider an application of (cut) as shown in proof $\Pi$ from Definition 2. If the cut-formula $\phi$ is SE-principal in $\mathrm{X} \vdash \phi$ and $\phi \vdash \mathrm{Y}$, then an SE-principal move is done otherwise a parametric move is done.

SE-principal moves There are three cases:

Case 1: $\mathrm{X} \vdash \mathrm{Y}$ is $\mathrm{X} \vdash \phi$ [resp. $\phi \vdash \mathrm{Y}$ ]. Then, $\Pi$ is transformed into $\Pi_{1}$ [resp. $\Pi_{2}$ ]. Case 2: $\phi$ is not a name. The treatment of the similar case in [Wan98] (see also [Bel82]) applies except that one has to take into account the display postulate inferences. For instance, when $\phi=\neg \psi$ the proof fragment below left is transformed into the proof fragment below right:

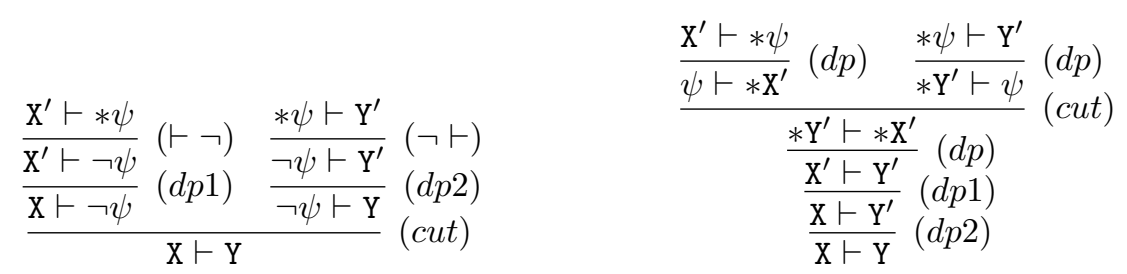

Observe that, in the transformed proof, the cut-formula $\psi$ has $d g(\psi)<d g(\phi)$. Case 3: $\phi$ is the name $i$. Then $\Pi$ is as shown below left and is transformed into the proof $\Pi^{\prime}$ shown below right:

$$
\frac{\frac{\mathrm{i} \vdash \neq * \mathrm{X}^{\prime}}{\frac{\mathrm{X}^{\prime} \vdash \mathrm{i}}{\mathrm{X} \vdash \mathrm{i}}(d p 1)}(\vdash) \frac{\mathrm{Y}^{\prime} \vdash \mathrm{i}}{\mathrm{i} \vdash \bullet \neq * \mathrm{Y}^{\prime}}(\mathrm{i} \vdash)}{\mathrm{X} \vdash \mathrm{Y}}(d p 2)
$$$$
\begin{aligned}
& \frac{\mathrm{i} \vdash \bullet * \mathrm{X}^{\prime} \quad \mathrm{Y}^{\prime} \vdash \mathrm{i}}{\mathrm{Y}^{\prime} \vdash * \bullet \neq \mathrm{X}^{\prime}}(\text { cut }) \\
& \frac{\frac{\mathrm{X}^{\prime} \vdash \bullet * \mathrm{Y}^{\prime}}{\mathrm{X} \vdash \bullet * \mathrm{Y}^{\prime}}}{\frac{\mathrm{X} \vdash \mathrm{Y}}{(d p)}}(d p 1) \\
&
\end{aligned}
$$

It is obvious that $(d p)$ moves do not alter a sequent in any significant way. So let us consider only significant (i.e. non $(d p)$ ) inferences. In Case 3 , the degree of the cut-formula in $\Pi^{\prime}$ equals the degree of the cut-formula $\phi$ but the number of significant inferences in $\Pi^{\prime}$ is less than in $\Pi$. In the proof of the strong normalisation theorem (see [DG98a]), the measure on the size of proofs counts only the number of significant inferences (and this measure decreases when required). Indeed, we implicitly consider as identical the sequents that are structurally equivalent (i.e. interderivable by using only the display postulates from Figure 2).

Parametric moves The parametric moves can be viewed simply as non SEprincipal moves. Suppose $\phi$ is not SE-principal in the inference ending in $\mathrm{X} \vdash \phi$ from Definition 2 in proof $\Pi$ (the other case is analogous). Viewing the congruence class $Q_{\phi}$ of this occurrence of $\phi$ as a tree, if the tree $Q_{\phi}$ contains an application of cut, then no reduction is performed and we instead consider one of the applications of cut above $\mathrm{X} \vdash \phi$ for reduction. Thus the shown application of cut from $\Pi$ is not subject to reduction at this stage. If the tree contains no application of cut, then for each path in $Q_{\phi}$ consider $\phi_{u}$ the uppermost member 
of $Q_{\phi}$ on the path and let $\mathcal{I}_{u}$ be the inference ending in the sequent $s$ which contains $\phi_{u}$.

Case (i): $\phi_{u}$ is principal in $\mathcal{I}_{u}$. So $\phi_{u}$ is the entire succedent of $s$. We cut with $\Pi_{2}$ and replace every occurrence of $\phi$ below $\phi_{u}$ in the path by Y.

Case (ii): $\phi_{u}$ is not principal in $\mathcal{I}_{u}$. Then, w.r.t. $\mathcal{I}, \phi_{u}$ is congruent only to itself so we just replace every occurrence of $\phi$ below $\phi_{u}$ in the path by Y. $\Pi_{2}$ is deleted.

Primitive Reduction. The result of simultaneously carrying out these operations for every path of occurrences of $\phi$ in $\Pi_{1}$ and removing the initial occurrence of $\mathrm{X} \vdash \mathrm{Y}$ is by definition a primitive reduction.

The treatment of the last two cases is exactly what is done in [Wan98] (see also [Bel82]). Fortunately, by close examination of Case (i) and Case (ii), it also works when $\phi$ is a name. Indeed, as mentioned previously, the two occurrences of $i$ in both rules $(i \vdash)$ and $(\vdash i)$ are not congruent by definition, and therefore, there is no need to treat the case $\phi=i$ separately.

The reduction process does not systematically remove the uppermost cut (this is just a particular case) and not all the cuts in a proof are necessarily subject to primitive reduction. For any non cut-free proof $\Pi$, we write $\Pi^{\prime}<\Pi$ to denote that $\Pi^{\prime}$ is obtained from $\Pi$ by application of a primitive reduction.

Theorem 6. (strong normalization) The relation $<$ on proofs of $\delta$ is wellfounded (no infinite decreasing chains) and the terminal proofs (those that cannot be reduced) are cut-free.

These proofs are impervious to additional structural rules obeying (C1)-(C7). The full proof of Theorem 6 can be found in [DG98a].

\section{Properly Displayable Nominal Tense Logics}

The aim of this section is to identify classes of properly displayable nominal tense logics by adapting developments from [Bla90,Kra96]. In what follows, we write $\delta+\mathcal{R}$ to denote the display calculus $\delta$ augmented with the set $\mathcal{R}$ of rules.

Definition 3. Logic $\mathcal{L}=\langle\operatorname{NTL}(H, G), \mathcal{C}\rangle$ is properly displayable $\stackrel{\text { def }}{\Leftrightarrow}$ there is a display calculus $\delta \stackrel{\text { def }}{=} \delta$ MNTL $+\mathcal{R}$ such that $\mathcal{R}$ is a set of structural rules satisfying (C2)-(Cr) and for any $\phi \in \operatorname{NTL}(H, G), \phi$ is $\mathcal{L}$-valid iff $I \vdash \phi$ is derivable in $\delta$.

Theorem 7. Every properly displayable logic has a cut-free display calculus.

Indeed, by Theorem $6, \delta$ MNTL $+\mathcal{R}$ admits a (strongly normalising) cutelimination theorem since all the rules in $\mathcal{R}$ satisfy the conditions (C2)-(C7).

Sahlqvist tense formulae are useful to study the nominal tense logics characterized by classes of frames modally definable by such formulae. A formula is positive [resp. negative] $\stackrel{\text { def }}{\Leftrightarrow}$ every propositional variable occurs under an even [resp. odd] number of negation symbols (when $\phi_{1} \Rightarrow \phi_{2}$ is treated as $\neg \phi_{1} \vee \phi_{2}$ ). A simple Sahlqvist tense formula in $\operatorname{TL}(H, G)$ is an implication $\phi \Rightarrow \psi$ such that $\psi$ 
is positive and $\phi$ is built up from negative formulae, formulae without occurrences of atomic propositions and formulae of the form $\sigma \mathrm{p}$ with $\sigma$ a universal modality and $\mathrm{p} \in \mathrm{PRP}$ using only $\wedge, \vee$ and the existential modalities; see e.g. [Rij93]. A Sahlqvist tense formula is a conjunction of formulae of the form $\sigma(\phi \Rightarrow \psi)$ where $\sigma$ is a universal modality and $\phi \Rightarrow \psi$ is a simple Sahlqvist tense formula.

Theorem 8. Let $\phi$ be a Sahlqvist tense formula and let $\vdash \stackrel{\text { def }}{=} \vdash_{\text {MNTL }}+\phi$. Then, any $\psi \in \operatorname{NTL}(H, G)$ is $\operatorname{NTL}_{\phi}$-valid iff $\vdash \psi$.

Theorem 8 does not follow from Sahlqvist's Theorem [Sah75] since NTL ${ }_{\phi}$ is known to be non canonical for any Sahlqvist tense formula $\phi$ where $\{\mathcal{F} \in F r$ : $\mathcal{F}=\phi\}$ contains a frame with a reflexive world and a frame with an irreflexive world [Bla90, Proof of Theorem 4.3.1]. For instance take $\phi$ to be $\mathrm{p} \Rightarrow \mathrm{p}$. That is, there is no (single canonical) $\mathrm{NTL}_{\phi}$-model $\mathcal{M}=(W, R, m)$ such that for every $\vdash$-consistent set $X$ there is $w \in W$ such that for all $\psi \in X, \mathcal{M}, w \models \psi$.

Theorem 9. Any logic $\mathcal{L}=\langle\operatorname{NTL}(H, G), \mathcal{C}\rangle$ where $\mathcal{C}=\{\mathcal{F} \in F r: \mathcal{F} \equiv \phi\}$ for some conjunction $\phi$ of primitive formulae in $\mathrm{TL}(G, H)$ is properly displayable.

The primitive formulae in Theorem 9 do not contain the difference operator. Another class of properly displayable nominal tense logics can be identified.

Theorem 10. Let $\mathcal{L}_{\neq}=\langle\mathrm{TL}(H, G,[\neq]), \mathcal{C}\rangle$ and let $\gamma$ be a conjunction of primitive formulae over the language $\operatorname{TL}(H, G,[\neq])$ such that $\vdash_{\neq}+\gamma$ axiomatizes $\mathcal{L}_{\neq}$and $\mathcal{C}$ is closed under disjoint unions and isomorphic copies. Then the logic $\mathcal{L}=\langle\operatorname{NTL}(H, G), \mathcal{C}\rangle$, is properly displayable.

The irreflexivity rule is not present in $\vdash_{\neq}+\gamma$. However, unlike Theorem 9 , the primitive axioms in Theorem 10 may contain the difference operator.

Proof. (sketch) Since struc $(\delta$ MNTL $)$ contains $\bullet \neq$, we first transform $\gamma$ into a collection $\mathcal{R}_{\gamma}$ of structural rules over struc( $\delta$ MNTL) using Kracht's method [Kra96]. This gives a display calculus $\delta \mathcal{L}_{\neq} \stackrel{\text { def }}{=} \delta \mathrm{MTL}_{\neq}+\mathcal{R}_{\gamma}$ where $\delta \mathrm{MTL}_{\neq}$is the display calculus for $\mathrm{MTL}_{\neq}$. Actually, $\delta \mathrm{MTL}_{\neq}$can be defined from $\delta$ MNTL by: considering the same set of structural connectives but building up the structures from $\operatorname{TL}(H, G,[\neq])$ instead of $\operatorname{TL}(H, G)$; deleting the fundamental axioms of the form $i \vdash i$ and the rules $(i \vdash)$ and $(\vdash \mathrm{i})$; and adding the rules below:

$$
\frac{\phi \vdash \mathrm{X}}{[\neq] \phi \vdash \bullet \neq \mathrm{X}}([\neq] \vdash) \quad \frac{\mathrm{X} \vdash \bullet_{\neq} \phi}{\mathrm{X} \vdash[\neq] \phi}(\vdash[\neq])
$$

Since $\delta \mathcal{L}_{\neq}$obeys $(\mathrm{C} 1)-(\mathrm{C} 8)$, it enjoys cut-elimination. To show that $\delta \mathcal{L} \stackrel{\text { def }}{=} \delta$ MNTL + $\mathcal{R}_{\gamma}$ properly displays $\mathcal{L}$, let $\operatorname{struc}\left(\delta \mathcal{L}_{\neq}\right)$be the set of structures involved in $\delta \mathcal{L}_{\neq}$ and define a partial function $g: \operatorname{struc}\left(\delta \mathcal{L}_{\neq}\right) \rightarrow \operatorname{struc}(\delta$ MNTL $)$ as follows:

- $g(\mathrm{X})$ is undefined if $\mathrm{X}$ contains some occurrences of $[\neq] \psi$ where $\psi$ is not of the form $\neg \mathrm{p}_{2 \times k+1}$ for $k \in \omega$; otherwise

- $g$ is homomorphic for the Boolean connectives, for $H$ and for $G$; 
- for any $k \in \omega, g\left(\mathrm{p}_{2 \times k+1}\right) \stackrel{\text { def }}{=} g\left([\neq] \neg \mathrm{p}_{2 \times k+1}\right) \stackrel{\text { def }}{=} \mathrm{i}_{k}$ and $g\left(\mathrm{p}_{2 \times k}\right) \stackrel{\text { def }}{=} \mathrm{p}_{k}$;

$-g(\perp) \stackrel{\text { def }}{=} \perp ; g(\top) \stackrel{\text { def }}{=} \mathrm{\top}$;

- $g$ is homomorphic for the structural connectives and $g(I) \stackrel{\text { def }}{=} I$.

Let $\phi$ be a formula of $\operatorname{NTL}(G, H)$.

- (soundness) If $I \vdash \phi$ has a cut-free proof in $\delta \mathcal{L}$, then $s(\phi)$ is $\mathcal{L}_{\neq}$-valid (where $s$ is from the proof of Theorem 3 and we use the closure properties of $\mathcal{C}$ ). We also have that $\phi$ is $\mathcal{L}$-valid iff $s(\phi)$ is $\mathcal{L}_{\neq^{-}}$-valid. Hence, $\phi$ is $\mathcal{L}$-valid. Note that in general $\phi \neq s(\phi)$ !

- (completeness) We must show that if $\phi$ is $\mathcal{L}$-valid, then $I \vdash \phi$ has a cut-free proof in $\delta \mathcal{L}$. The proof contains five parts: (1) if $\phi$ is $\mathcal{L}$-valid, then $a(\phi)$ is

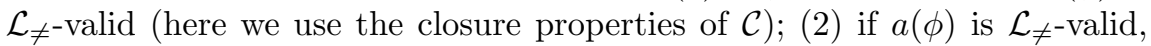
then $I \vdash a(\phi)$ has a cut-free proof in $\delta \mathcal{L}_{\neq} ;(3)$ if $I \vdash a(\phi)$ has a cut-free proof in $\delta \mathcal{L}_{\neq}$, then $I \vdash g(a(\phi))$ has a cut-free proof in $\delta \mathcal{L}$; $(4) I \vdash g(a(\phi))$ has a cut-free proof in $\delta \mathcal{L}$ iff $I \vdash \phi$ has a cut-free proof in $\delta \mathcal{L} ;(5)$ hence, if $\phi$ is $\mathcal{L}$-valid, then $I \vdash \phi$ has a cut-free proof in $\delta \mathcal{L}$ (see the details in [DG98a]).

The proof of Theorem 10 is very informative since for instance, it also shows that any formula $\phi \in \operatorname{NTL}(H, G)$ is MNTL-valid iff $I \vdash \phi$ has a cut-free proof in $\delta$ MNTL. Unlike the proof of Theorem 4 , the $(\vdash \mathbf{i})$-rule is used. The rules $(i \vdash)$ and $(\vdash \mathrm{i})$ are obviously equivalent to the reversible rule below:

$$
\frac{\mathrm{X} \vdash \mathrm{i}}{\mathrm{i} \vdash \bullet * \mathrm{X}}
$$

Hence, as in the case with the display postulates, or indeed any reversible rule, backward proof search may enter loops. However, all is not lost, for the proof of Theorem 10 also yields

Corollary 1. In a backward proof attempt, if we apply $(\mathrm{i} \vdash)$ [resp. $(\vdash \mathrm{i})$ ], giving rise to some name $\mathrm{i}$ in the premiss, then we do not need to apply $(\vdash \mathrm{i})$ [resp. $(\mathrm{i} \vdash)]$ to this name in the rest of the backward proof search.

\section{Concluding Remarks}

To define cut-free display calculi for nominal tense logics, we have extended Wansing's strong normalization theorem [Wan98] to any reasonable extension of $\delta$ MNTL. Although $\delta$ MNTL does not satisfy (C8), the proof in Section 4 provides a new condition $\left(\mathrm{C}^{\prime} 8\right)$ (see the appendix).

Are the classes of properly displayable nominal tense logics characterised by Theorem 9 and Theorem 10 really different? One solution is to characterize the class of Sahlqvist tense formulae $\phi$ such that $\vdash_{\neq}+\phi$ axiomatizes $\langle\mathrm{TL}(H, G,[\neq$ ]), $\{\mathcal{F}: \mathcal{F}=\phi\}\rangle$. This is roughly equivalent to knowing when the irreflexivity rule is superfluous (see e.g. [Ven93]). How to define structural rules in DL from axioms containing names? 
Kracht and Wolter [KW97] show how to eliminate the difference operator by means of a pair of tense operators. Unfortunately, one operator must satisfy the Gödel-Lob axiom G, which is not Sahlqvist. Using our recent work on cut-free display calculi for such "second-order" modal logics [DG99], we may be able to design yet another display calculus for nominal tense logics.

\section{References}

[BD97] Ph. Balbiani and S. Demri. Prefixed tableaux systems for modal logics with enriched languages. In IJCAI-15, pages 190-195. Morgan Kaufmann, 1997.

[Bel82] N. Belnap. Display logic. Journal of Philosophical Logic, 11:375-417, 1982.

[Bla90] P. Blackburn. Nominal Tense Logic and Other Sorted Intensional Frameworks. PhD thesis, University of Edinburgh, Edinburgh, 1990.

[Bla93] P. Blackburn. Nominal tense logic. NDJFL, 34(1):56-83, 1993.

[Bla98] P. Blackburn. Internalizing labeled deduction. Technical Report 102, Computerlinguistik, Universität des Saarlandes, 1998.

[Dem99] S. Demri. Sequent Calculi for Nominal Tense Logics: a step toward mechanization?. In this volume.

[DG98a] S. Demri and R. Goré. Cut-free display calculi for nominal tense logics. TRARP-07-98, A.R.P., A.N.U., 1998. http://arp.anu.edu.au/.

[DG99] S. Demri and R. Goré. Theoremhood Preserving Maps as a Characterisation of Cut Elimination for Provability Logics. TR-ARP-??-99, forthcoming 1999.

[dM94] M. d'Agostino and M. Mondadori. The taming of the cut. Classical refutations with analytic cut. J. of Logic and Computation, 4(3):285-319, 1994.

[Gab81] D. Gabbay. An irreflexivity lemma with applications to axiomatization of conditions on tense frames. In U. Mönnich, editor, Aspects of Philosophical Logic, pages 67-89. Reidel, 1981.

[GG93] G. Gargov and V. Goranko. Modal logic with names. J. of Philosophical Logic, 22(6):607-636, 1993.

[Gor95] R. Goré. Intuitionistic logic redisplayed. TR-ARP-1-95, ARP, ANU, 1995.

[Gor98] R. Goré. Substructural logics on display. LJIGPL, 6(3):451-504, 1998.

[GT75] R. Goldblatt and S. Thomason. Axiomatic classes in propositional modal logic. In Algebra and Logic, LNM 450:163-173, 1975, Springer-Verlag.

[Kon97] B. Konikowska. A logic for reasoning about relative similarity. Studia Logica, 58(1):185-226, 1997.

[Koy92] R. Koymans. Specifying message passing and time-critical systems with temporal logic. LNCS 651, Springer-Verlag, 1992.

[Kra96] M. Kracht. Power and weakness of the modal display calculus. In H. Wansing, editor, Proof theory of modal logic, pages 93-121. Kluwer, 1996.

[KW97] M. Kracht and F. Wolter. Simulation and transfer results in modal logic - A survey. Studia Logica, 59:149-1997, 1997.

[Orło84] E. Orłowska. Logic of indiscernibility relations. In 5th Symposium on Computation Theory, pages 177-186. LNCS 208, Springer-Verlag, 1984.

[PT85] S. Passy and T. Tinchev. PDL with data constants. IPL, 20:35-41, 1985.

[PT91] S. Passy and T. Tinchev. An essay in combinatory dynamic logic. Information and Computation, 93:263-332, 1991.

[Rij92] M. de Rijke. The modal logic of inequality. JSL, 57(2):566-584, 1992.

[Rij93] M. de Rijke. Extending modal logic. PhD thesis, ILLC, Amsterdam, 1993. 
[Sah75] H. Sahlqvist. Completeness and correspondence in the first and second order semantics for modal logics. In S. Kanger, editor, 3rd Scandinavian Logic Symposium, pages 110-143. North Holland, 1975.

[Seg81] K. Segerberg. A note on the logic of elsewhere. Theoria, 47:183-187, 1981.

[TS96] A. Troelstra and H. Schwichtenberg. Basic Proof Theory. Cambridge Tracts in Theoretical Computer Science 43. Cambridge University Press, 1996.

[Ven93] Y. Venema. Derivation rules as anti-axioms in modal logic. JSL, 58(3):1003$1034,1993$.

[Wan94] H. Wansing. Sequent calculi for normal modal propositional logics. J. of Logic and Computation, 4(2):125-142, 1994.

[Wan98] H. Wansing. Displaying Modal Logic, volume 3 of Trends in Logic. Kluwer Academic Publishers, Dordrecht, 1998. 


\section{Appendix: Belnap's Conditions.}

For every sequent rule Belnap [Bel82, page 388] first defines the following notions: in an application Inf of a sequent rule $(\rho)$, "constituents occurring as part of occurrences of structures assigned to structure-variables are defined to be parameters of Inf ; all other constituents are defined as nonparametric, including those assigned to formula-variables. Constituents occupying similar positions in occurrences of structures assigned to the same structure-variable are defined as congruent in Inf". The eight (actually seven) conditions shown below are from [Kra96]:

(C1) Each formula which is a constituent of some premiss of a rule $\rho$ is a subformula of some formula in the conclusion of $\rho$.

(C2) Congruent parameters are occurrences of the same structure.

(C3) Each parameter is congruent to at most one constituent in the conclusion. Equivalently, no two constituents of the conclusion are congruent to each other.

(C4) Congruent parameters are either all antecedent parts or all succedent parts of their respective sequent.

(C5) If a formula is non-parametric in the conclusion of a rule $\rho$, it is either the entire antecedent, or the entire succedent. Such a formula is called a principal formula.

(C6/7) Each rule is closed under simultaneous substitution of arbitrary structures for congruent parameters.

(C8) If there are inference rules $\rho_{1}$ and $\rho_{2}$ with respective conclusions $\mathrm{X} \vdash \phi$ and $\phi \vdash \mathrm{Y}$ with $\phi$ principal in both inferences (in the sense of C5), and if (cut) is applied to yield $\mathrm{X} \vdash \mathrm{Y}$ then, either $\mathrm{X} \vdash \mathrm{Y}$ is identical to $\mathrm{X} \vdash \phi$ or to $\phi \vdash \mathrm{Y}$; or it is possible to pass from the premisses of $\rho_{1}$ and $\rho_{2}$ to $\mathrm{X} \vdash \mathrm{Y}$ by means of inferences falling under (cut) where the cut-formula is always a proper subformula of $\phi$. If $\phi$ satisfies the "if" part of this condition it is known as a "matching principal constituent".

\section{Our new condition $\left(\mathbf{C 8}^{\prime}\right)$}

$\left(\mathbf{C 8}^{\prime}\right)$ There exist a non-empty set $S$ with $<$ a well-founded ordering on $S$ and a map $d g:$ For $\rightarrow S$ such that if there are inferences $\mathcal{I}_{1}$ and $\mathcal{I}_{2}$ with respective conclusions $\mathrm{X} \vdash \phi$ and $\phi \vdash \mathrm{Y}$ with $\phi$ SE-principal in both inferences, and if cut is applied to obtain $\mathrm{X} \vdash \mathrm{Y}$, then

- either $\mathrm{X} \vdash \mathrm{Y}$ is identical to one of $\mathrm{X} \vdash \phi$ and $\phi \vdash \mathrm{Y}$;

- or there is a derivation of $\mathrm{X} \vdash \mathrm{Y}$ from the premisses of $\mathcal{I}_{1}$ and $\mathcal{I}_{2}$ in which every cut-formula $\psi$ of any application of cut satisfies $d g(\psi)<d g(\phi)$;

- or there is a derivation of $\mathrm{X} \vdash \mathrm{Y}$ from the premisses of $\mathcal{I}_{1}$ and $\mathcal{I}_{2}$ in which every cut-formula $\psi$ of any application of cut satisfies $d g(\psi)=d g(\phi)$ and in that derivation every inference, except possibly one, falls under an invertible structural rule with a single premiss. 